\title{
Recombinant Human Thrombomodulin for Pneumonia-Induced Severe Acute Respiratory Distress Syndrome Complicated by Disseminated Intravascular Coagulation in Children: A feasibility study
}

Naoyuki Hirata ( $\square$ naohirata@mac.com )

Sapporo Medical University School of Medicine https://orcid.org/0000-0002-3776-8115

Dong Tien Ngo

Vietnam National Children's Hospital

Phuc Huu Phan

Vietnam National Children's Hospital

Akira Ainai

National Institute of Infectious Diseases

Thuy Thi Bich Phung

Vietnam National Children's Hospital

Ta Anh Tuan

Vietnam National Children's Hospital

Tran Minh Dien

Vietnam National Children's Hospital

Jin Takasaki

National Center for Global Health and Medicine

Shoji Kawachi

Teikyo Daigaku"

Noriko Nakajima

National Institute of Infectious Diseases

Le Thanh Hai

Vietnam National Children's Hospital

Research article

Keywords: pediatric acute respiratory distress syndrome, disseminated intravascular coagulation, recombinant soluble human thrombomodulin 
Posted Date: October 31st, 2019

DOI: https://doi.org/10.21203/rs.2.16658/v1

License: (c) (i) This work is licensed under a Creative Commons Attribution 4.0 International License. Read Full License 


\section{Abstract}

Background Recombinant human soluble thrombomodulin (rTM) has been used to treat disseminated intravascular coagulation (DIC). Recent studies have shown the efficacy of rTM through its antiinflammatory effects for treatment of adults with acute respiratory distress syndrome (ARDS). However, the safety and efficacy of rTM in children with severe ARDS complicated by DIC have not been reported. In this study, we investigated the feasibility of using rTM for the treatment of pneumonia-induced severe ARDS complicated by DIC in children.

Methods Six children (age: median 10 month-old) with pneumonia-induced severe ARDS complicated by DIC were enrolled in this feasibility study. rTM $(380 \mathrm{U} / \mathrm{kg})$ was administered for a maximum of 6 days, in addition to conventional therapies including cardiopulmonary support, antibiotics and/or antivirus drugs administration, steroid administration and intravenous immunoglobulin after diagnosis of severe ARDS complicated by DIC. After administration of rTM, we measured changes in the plasma TM concentration and evaluated the clinical course, status of DIC and ARDS, and other laboratory findings, including levels of cytokines, chemokines, and biomarkers.

Results In all six children, the plasma concentration of TM increased and DIC scores decreased after administration of rTM. Four of the six children recovered from the severe ARDS complicated by DIC after treatment in the pediatric intensive care unit, and were discharged from the hospital with no complications. In surviving children, levels of soluble receptors for advanced glycation end products, interleukin-6, interleukin-8 and monocyte chemotactic protein-1 decreased after administration of rTM.

Conclusions The rTM administration is feasible as a therapeutic strategy for children over 2 months with pneumonia-induced severe ARDS complicated by DIC.

\section{Background}

Pneumonia is a leading infectious cause of hospitalizations and death among children globally [1]. Pneumonia can induce acute respiratory distress syndrome (ARDS) [2,3], and disseminated intravascular coagulopathy (DIC) can develop in patients with severe ARDS [4]. Severe ARDS complicated by DIC is a life-threatening condition and is associated with a poor prognosis, indicating the need for early treatment [4].

Recombinant human soluble thrombomodulin (rTM) was primarily developed in Japan to treat DIC [5]. Based on its safety and efficacy, rTM is now administered to treat children with DIC [6, 7]. Recently, several clinical studies have been conducted to evaluate the efficacy of rTM as an adjunctive antiinflammatory agent for various severe inflammatory conditions [8, 9]. Adjunctive rTM administration may be effective for ARDS complicated by DIC and sepsis in adult patients [9, 10]; however, the detailed clinical effects of rTM in children with severe ARDS complicated by DIC have not yet been clarified. This study aims to prospectively evaluate the feasibility of the use of rTM for the treatment of children with severe ARDS complicated by DIC. We examined not only the change of plasma levels of thrombomodulin 
(TM) after rTM administration but also that of cytokines/chemokines and biomarkers which were associated with systemic inflammation and lung injury.

\section{Methods}

\section{Patients and case definitions}

This prospective feasibility study was carried out from January 2016 to May 2018 at the PICU in Vietnam National Children's Hospital, Hanoi, Vietnam. Children who met the following inclusion criteria within 72 hours of PICU admission were enrolled: (1) admission to the PICU with infectious pneumonia; (2) age of 1 month to 15 years; (3) DIC scores defined by the International Society of Thrombosis and Haemostasis $\left(\right.$ ISTH) $\geq 5$; (4) $\mathrm{PaO}_{2} / \mathrm{FiO}_{2}$ ratio (P/F ratio) $\leq 100 \mathrm{~mm} \mathrm{Hg}$ (based on the Berlin definition of 2012) [11]; (5) No severe left ventricular dysfunction; (6) Chest X-ray showing an abnormal shadow (consolidation, ground-glass opacity, or nodular shadow); and (7) Written informed consent prior to initiation of any study procedures, obtained from the patient's parents. Patients with severe renal dysfunction (creatinine clearance $<10 \mathrm{~mL} / \mathrm{min}$ ) or bleeding symptoms, or those whom the treating doctor deemed ineligible for any reason, were excluded from this study.

\section{rTM administration}

Similarly to the administration of rTM to adults, rTM $(380 \mathrm{U} / \mathrm{kg})$ was administered for a maximum of 6 consecutive days via intravenous drip infusion for 30 minutes. All necessary treatments performed by PICU doctors were acceptable, except administration or co-administration of anticoagulants, thrombolytic agents, and platelet aggregation inhibitors. Blood samples were taken on the day of admission to the PICU (day X), before the first administration of rTM, and on a days after day $X$ (day $X+a$ ). Blood samples were used to evaluate plasma concentrations of TM, cytokines/chemokines, biomarkers, blood cell counts, blood chemistry and DIC scores. The rTM administration was discontinued in case that patients developed severe renal dysfunction or bleeding in vital organs. We observed the clinical time course including vital signs and physical examination findings, bleeding symptoms, P/F ratio, oxygenation index, ventilator settings, and radiographic findings.

\section{Microbiological tests}

Blood and tracheal lavage fluid (TLF) were collected on the day of admission to the PICU. Microbiological cultures were prepared according to standard microbiological procedures. To detect and differentiate up to 25 pathogenic microbial DNA types in blood samples, the LightCycler SeptiFast Test (Roche Diagnostics $\mathrm{GmbH}$, Mannheim, Germany) was used [12]. Total nucleic acids from the TLF samples were extracted with a MagNA Pure LC total nucleic acid isolation kit using a Roche MagNA Pure LC instrument according to the manufacturer's instructions (Roche Diagnostics). The extracts were tested by multiplex real-time reverse transcriptase (rRT) polymerase chain reaction (PCR) (rRT-PCR) using the FTD 
Respiratory Pathogens 33 Kit according to the manufacturer's instructions (Fast Track Diagnostics, Junglinster, Luxembourg) to screen 33 kinds of respiratory pathogens and by an in-house developed conventional single-target rRT-PCR and real-time PCR (rPCR) to detect genomes of cytomegalovirus (CMV), human immunodeficiency virus (HIV), and varicella zoster virus (VZV).

\section{TM and biomarker assay}

The plasma concentration of TM, soluble receptor of advanced glycation end products (SRAGE), pulmonary surfactant protein D (SP-D), angiopoietin-2 (Ang-2) and high-mobility group box 1 (HMGB1) were measured using enzyme-linked immunosorbent assay (ELISA) kits and a microplate reader (Multiskan FC; Thermo Fisher Scientific K. K., Tokyo, Japan). ELISA kits for TM, sRAGE, SP-D and Ang-2 were purchased from R\&D Systems (Tokyo, Japan) and for HMGB-1 from Shino-Test Corporation (Tokyo, Japan). All samples were run in duplicate and the average concentrations were used for statistical analysis.

\section{Cytokine and chemokine assay}

The levels of cytokines/chemokines in the plasma were measured using the Human Cytokine Magnetic 25-Plex Panel Kit (Invitrogen, Carlsbad, CA, USA) on a Magpix ${ }^{\circledR}$ system (Merck Vietnam Co., Ltd, Vietnam). All samples were run in duplicate and the average concentrations were used for statistical analysis.

\section{Statistical analysis}

DIC scores were shown as medians with the interquartile range (IQR), and the groups (day X and day $X+6$ ) were compared using the Wilcoxon signed rank test. $P<0.05$ was considered statistically significant. Data were statistically analyzed using Prism 5.0 for Mac (GraphPad Software Inc., La Jolla, CA, USA) with a two-tailed hypothesis.

\section{Results}

\section{Patients}

Six children fulfilled the inclusion criteria of having severe ARDS and DIC. Although more than one virus genome was detected in each patient, the major causative pathogen of pneumonia in each case was estimated to be respiratory syncytial virus, tuberculosis, influenza A/H1N1pdm, Pneumocystis jirovecii / CMV, CMV and VZV, respectively (Table 1). No pathogenic microbial DNA was detected in blood samples, suggesting no bacteremia and fungiemia. Case 3 (1y5M) and Case 4(3M) had past history of recurrent pneumonia. Case 4 was HIV positive. The other children had no significant past history. 


\section{Safty of rTM administartion}

Each patient except case 3 was administered rTM for 6 consecutive days after enrolment in this study. In case 3 , rTM was discontinued on day $X+3$, because slightly bloody urine was detected after the fourth administration of rTM. The other cases showed no bleeding or other complications throughout the study. We did not find any adverse effects of rTM on liver or renal function during rTM administration (Table 2). We measured the plasma concentration of TM before rTM administration on day $\mathrm{X}$ and a days after administration (on day $\mathrm{X}+\mathrm{a}$ ). On the days after rTM administration, the plasma concentration of TM increased in all patients (Figure 1). DIC scores decreased significantly after administration of rTM $(P<$ 0.05) (Figure 2).

In cases 1,3 , and 4 , the status of ARDS, including the P/F ratio and oxygenation index, improved and they were successfully extubated within a week after administration of rTM (Table 3). Case 2 required more time for improvement in the respiratory conditions due to tuberculous pneumonia, but this patient was later successfully extubated and survived [13]. Two patients, cases 5 and 6 , died from their respiratory conditions that had not fully resolved (Table 3), although their DIC scores improved during administration of rTM. Case 5 developed severe hypoxia from respiratory failure occurred 7 days after completion of rTM administration (day X+12). In case 6, her respiratory status deteriorated after multiple drug-resistant Acinetobacter baumannii was cultured from TLF 5 days after completion of rTM administration.

\section{Biomarkers and inflammatory cytokines / chemokines}

Laboratory findings revealed that SRAGE, which is a biomarker of lung epithelial injury, decreased after administration of rTM in all 6 children (Figure 3). Plasma levels of interleukin (IL) 6 (Figure 4), and IL-8 (Figure 5) also decreased in all patients after administration of rTM, except in case 5. Monocyte chemotactic protein-1 (MCP-1) is a member of the chemokine family, and regulates trafficking of monocytes from bone marrow to inflamed tissue in response to inflammatory signals [14]. Although plasma MCP-1 levels in the surviving children (except on day $\mathrm{X}+5$ in case 4 ) decreased after rTM administration, those in the children who died increased (Figure 6). However, there was variability in changes in other biomarkers, such as HMGB-1 (Additional file 1), SP-D (Additional file 2) and Ang2 (Additional file 3), and cytokines/chemokines, such as interferon-gamma (IFN- $\gamma$ ) (Additional file 4), IL-10 (Additional file 5), and interferon- $\gamma$-inducible protein-10 (IP-10) (Additional file 6), among the patients.

\section{Discussion}

This prospective feasibility study showed that rTM administration increased the plasma concentration of TM and decreased DIC scores in children with severe ARDS complicated by DIC. In all six children, SRAGE decreased rapidly after rTM administration. In surviving children, levels of IL-6, IL-8 and MCP-1 decreased after administration of rTM. Our study suggests that the feasibility of administration of rTM for the treatment of children with ARDS complicated by DIC. 
Previous studies in adults $[15,16]$ and children $[6,7]$ have shown the efficacy of rTM for DIC. We confirmed that rTM increased the plasma concentration of TM and DIC score decreased after rTM administration, consistent with previous studies. In adults, the effective concentration of rTM (TM alpha) is recommended to be more than $500 \mathrm{ng} / \mathrm{mL}$ at steady state [17]. Although few studies have focused on the plasma concentration of TM in children, Takeuchi et al. reported that the concentration of rTM (TM alpha) at steady state was 431 [278-727] $\mathrm{ng} / \mathrm{mL}$ when the same dose of rTM as in our study was used in children with hematological malignancy and DIC [18]. In our study, after administration of rTM the median of peak concentration of TM was 475 [IQR 273-585] ng/mL. In children, a higher dose of rTM may be required to achieve a TM level higher than $500 \mathrm{ng} / \mathrm{mL}$, and a higher concentration of TM may produce a better effect for not only the control of DIC but also of inflammation.

In patients who survived, the P/F ratio and oxygenation index improved after administration of rTM. Notably, sRAGE in all six children decreased after administration of rTM. sRAGE is a marker of lung epithelial injury [19], and has good diagnostic value for ARDS and is associated with the severity of lung injury, the degree of lung epithelial injury, impaired alveolar fluid clearance, and prognosis in ARDS [20, 21]. Based on these studies, administration of rTM may be related with improvement or attenuation of lung epithelial injury. However, respiratory conditions did not improve after rTM in the patients who died. Recently, Jabaudon et al. reported that a higher baseline level of SRAGE was associated with development of ARDS [22]. In this study, on day X (just before administration of rTM), the plasma concentrations of SRAGE in patients 5 and 6, who later died, were 4715 and $8501 \mathrm{pg} / \mathrm{mL}$, respectively, whereas the median concentration of sRAGE in the patients who survived was 1817 [IQR 1437-1904] $\mathrm{pg} / \mathrm{m}$. Thus, different degrees of damage in the lung epithelium in the patients who survived and those who did not survive may be involved in the different progress of ARDS and the subsequent outcomes.

In the patients who survived, rTM may have helped to improve the ARDS status via anti-inflammatory effects. Recently, the anti-inflammatory effects of rTM have been highlighted, and rTM may improve not only respiratory dysfunction but also mortality rates in patients with severe sepsis and DIC [8-10,23]. We previously published case reports on 3 of the patients who survived (cases 1,2 , and 4 ) in this study [13, 24]. Interestingly, MCP-1, which is one of the chemokines, decreased in 3 of the 4 patients who survived, but not in the patients who died, after administration of rTM. A previous study reported that a higher serum MCP-1 level was associated with a 10-fold higher risk of death in ARDS [25]. In addition to SRAGE, these biomarkers may be useful for predicting the development of ARDS and outcomes. In contrast, there was variability in changes in other biomarkers, such as several inflammatory biomarkers (HMGB-1, SP-D, Ang2) and cytokines/chemokines (IFN- - , IL-10, IP-10), which are associated with development of ARDS [26]. To determine the anti-inflammatory effects of rTM in patients with severe ARDS, further large studies with patients without rTM administration and with simultaneous measurement of multiple inflammatory biomarkers and cytokines/chemokines may be required.

\section{Limitations}


This study has several limitations. First, this feasibility study is not a randomized trial, and there were no control patients, who were treated without rTM. To validate the efficacy of rTM for children with severe ARDS complicated by DIC, a randomized controlled trial would be required. Second, we could not collect blood samples from the patients to evaluate the levels of biomarkers and cytokines/chemokines on the same days after administration of rTM because the children's conditions were different and the blood samples needed to be limited to avoid iatrogenic anemia.

\section{Conclusion}

In conclusion, we investigated the feasibility of rTM for the treatment of children with severe pneumoniainduced ARDS complicated by DIC. Our findings suggest that rTM improves DIC and may have beneficial effects in cases of pediatric ARDS via anti-inflammatory responses.

\section{List Of Abbreviations}

ARDS, acute respiratory distress syndrome; DIC, disseminated intravascular coagulation; OI, oxygenation index; $\mathrm{PF}$ ratio, $\mathrm{PaO}_{2} / \mathrm{FiO}_{2}$ ratio; $\mathrm{RT}$-PCR, real-time polymerase chain reaction; $\mathrm{rTM}$, recombinant human soluble thrombomodulin; HMGB-1, high mobility group box 1 ; SRAGE, soluble receptor for advanced glycation end products; PICU, pediatric intensive care unit; TLF, tracheal lavage fluid

\section{Declarations}

\section{Ethics approval and consent to participate}

This therapeutic protocol was approved by the biomedical research ethics committee of the National Hospital of Pediatrics Research Institute for Child Health (NHP-RICH, Hanoi, Vietnam) (reference number: NHP-RICH-15-008) and the ethical committee of the National Center for Global Health and Medicine (NCGM, Tokyo, Japan) (reference number: NCGM-G-001853-00).

The parents of all children provided written informed concent for participating to this study.

\section{Consent to publish}

The parents of all children provided written informed concent for the publication of this manuscript.

\section{Availability of data and materials}

The data supporting our findings can be found in the manuscript, tables and figures.

\section{Competing interests}


The authors state that they have no conflicts of interest.

\section{Funding}

The authors have not received money, gifts, or other compensation from any organization, institution, or business that may be affected financially by our publication.

\section{Authors' contributions}

DTN, PHP, TAT, DMT and HTL performed medical treatment for patients. SK, NN, JT and NH participated in the conception of the treatment protocol, and drafted the manuscript.AA and TTBP performed rRT-PCR assays and analyzed biomarkers of ARDS. All authors have read and approved the final manuscript.

\section{Acknowledgements}

This therapeutic strategy was supported by the Research Program on Emerging and Re-Emerging Infectious Diseases of the Agency for Medical Research and Development, Japan, under Grant Number JP18fk0108003.

\section{References}

1. Bhutta ZA, Das JK, Walker N, Rizvi A, et al. Interventions to address deaths from childhood pneumonia and diarrhoea equitably: what works and at what cost? Lancet 2013; 381:14171429

2. Dahlem P, van Aalderen WM, Bos AP. Pediatric acute lung injury. Paediatr Respir Rev 2007; 8:348362

3. Randolph AG. Management of acute lung injury and acute respiratory distress syndrome in children. Crit Care Med 2009; 37:2448-2454

4. Gando S, Kameue T, Matsuda N, et al. Systemic inflammation and disseminated intravascular coagulation in early stage of ALI and ARDS: Role of neutrophil and endothelial activation. Inflammation 2004; 28:237-244

5. Saito H, Maruyama I, Shimazaki S, et al. Efficacy and safety of recombinant human soluble thrombomodulin (ART-123) in disseminated intravascular coagulation: results of a phase III, randomized, double-blind clinical trial. J Thromb Haemost 2007; 5:31-41

6. Shirahata A, Mimuro J, Takahashi H, et al. Recombinant soluble human thrombomodulin (thrombomodulin alfa) in the treatment of neonatal disseminated intravascular coagulation. Eur $\mathrm{J}$ Pediatr 2014; 173:303-11

7. Shirahata A, Mimuro J, Takahashi $\mathrm{H}$, et al. Postmarketing Surveillance of Recombinant Human Soluble Thrombomodulin (Thrombomodulin a) in Pediatric Patients with Disseminated Intravascular 
Coagulation. Clin Appl Thromb Hemost 2014; 20:465-472.

8. Hayakawa M, Yamakawa K, Saito S, et al. Recombinant human soluble thrombomodulin and mortality in sepsis-induced disseminated intravascular coagulation. A multicenter retrospective study. Thromb Haemost 2016; 115:1157-1166

9. Ogawa Y, Yamakawa K, Ogura H, et al. Recombinant human soluble thrombomodulin improves mortality and respiratory dysfunction in patients with severe sepsis. J Trauma Acute Care Surg 2012; 72:1150-1157

10. Yoshihiro S, Sakuraya M, Hayakawa M, et al. Recombinant human-soluble thrombomodulin contributes to reduced mortality in sepsis patients with severe respiratory failure: a retrospective observational study using a multicenter dataset. Shock 2018; 51:174-179

11. Ranieri VM, Rubenfel GD, Thompson BT, et al. Acute respiratory syndrome: the Berlin definition. JAMA 2012; 307:2526-2533

12. Suberviola B, Márquez-López A, Castellanos-Ortega A, et al. Microbiological diagnosis of Sepsis: polymerase chain reaction system versus blood cultures. Am J Crit Care 2016; 25:68-75

13. Ngo DT, Phan PH, Kawachi S, et al. Tuberculous pneumonia-induced severe ARDS complicated with DIC in a female child: a case of successful treatment. BMC Infect Dis 2018; 18:294

14. Haller H, Bertram A, Nadrowitz F, et al. Monocyte chemoattractant protein-1 and kidney. Curr Opin Nephrol Hypertens 2016; 25:42-49

15. Aikawa N, Shimazaki S, Yamamoto Y, et al. Thrombomodulin alpha in the treatment of infectious patients complicated by disseminated intravascular coagulation: Subanalysis from the phase 3 trial. Shock $2011 ; 35: 349-354$

16. Yamakawa K, Ogura H, Fujimi S, et al. Recombinant human soluble thrombomodulin in sepsisinduced disseminated intravascular coagulation: A multicenter propensity score analysis. Intensive Care Med 2013; 39:644-652

17. Tsuruta K, Yamada Y, Serada M, et al. Model-based analysis of covariate effects on population pharmacokinetics of thrombomodulin alpha in patients with disseminated intravascular coagulation and normal subjects. J Clin Pharmacol 2011; 51:1276-1285

18. Takeuchi M, Tanoshima R, Miyagawa N, et al. Population pharmacokinetics of thrombomodulin alfa in pediatric patients with hematological malignancy and disseminated intravascular coagulation. Pediatr Blood Cancer 2017; 64. Doi: 10. 1002/pbc.26234

19. Uchida T, Shirasawa M, Ware LB, et al. Receptor for advanced glycation end-products is a marker of type I cell injury in acute lung injury. Am J Respir Crit Care Med 2006; 173:1008-1015

20. Calfee CS, Ware LB, Eisner MD, et al. Plasma receptor for advanced glycation end products and clinical outcomes in acute lung injury. Thorax 2008; 63:1083-1089

21. Jabaudon M, Blondonnet R, Roszyk L, et al. Solbule receptor for advanced glycation end-products predicts impaired alveolar fluid clearance in acute respiratory distress syndrome. Am J Respir Crit Care Med 2015; 192:191-199 
22. Jabaudon M, Berthelin P, Pranal T, et al. Receptor for advanced glycation end-products and ARDS prediction: a multicenter observational study. Sci Rep 2018; 8:2603

23. Yoshimura J, Yamakawa K, Ogura $\mathrm{H}$, et al. Benefit profile of recombinant human soluble thrombomodulin in sepsis-induced disseminated intravascular coagulation: a multicenter propensity score analysis. Crit Care 2015; 19:78

24. Phuc PH, Dong NT, Tuan TA, Kawachi S, et al. Successful treatment of pneumonia-induced severe ARDS complicated with DIC in two infants using recombinant human thrombomodulin. Integr Mol Med 2017; 4:1-5

25. Bautista $E$, Arcos $M$, Jimenez-Alvarez $L$, et al. Angiogenic and inflammatory markers in acute respiratory distress syndrome and renal injury associated to $A / H 1 N 1$ virus infection. Exp Mol Pathol 2013; 94:486-492

26. Fujishima S. Pathophysiology and biomarkers of acute respiratory distress syndrome. J Intensive Care $2014 ; 2: 32$

\section{Tables}

Table 1. The characteristics of patients

\begin{tabular}{|c|c|c|c|c|c|c|c|c|c|c|}
\hline & Gender & Age & $\begin{array}{l}\text { PRISM } \\
\text { III }\end{array}$ & $\begin{array}{c}\text { Lowest } \\
\mathrm{P} / \mathrm{F} \\
\text { ratio } \\
\end{array}$ & $\begin{array}{c}\text { Maximum } \\
\text { DIC } \\
\text { score } \\
\end{array}$ & $\begin{array}{c}\text { Pathogen in } \\
\text { TLF/NPA/blood } \\
\text { on PICU } \\
\text { admission } \\
\end{array}$ & $\begin{array}{c}\text { PICU } \\
\text { admission } \\
\text { day from } \\
\text { the onset }\end{array}$ & $\begin{array}{c}\text { LOS } \\
\text { In } \\
\text { PICU }\end{array}$ & $\begin{array}{c}\text { LOS } \\
\text { In } \\
\text { Hospital } \\
\end{array}$ & Outcome \\
\hline $\begin{array}{c}\text { Case } \\
1\end{array}$ & $\mathrm{~F}$ & $2 \mathrm{M}$ & 10 & 36 & 7 & RSV, CMV* & 6 & 10 & 21 & Alive \\
\hline $\begin{array}{c}\text { Case } \\
2\end{array}$ & $\mathrm{~F}$ & $2 \mathrm{Y} \square \mathrm{M}$ & 3 & 35 & 6 & TB, CMV* & 20 & 32 & 33 & Alive \\
\hline $\begin{array}{c}\text { Case } \\
3\end{array}$ & M & $1 \mathrm{Y} \square \mathrm{M}$ & 5 & 77 & 7 & $\begin{array}{c}\text { A/H1N1pdm, } \\
\text { CMV* }^{*}\end{array}$ & 8 & 13 & 27 & Alive \\
\hline $\begin{array}{c}\text { Case } \\
4\end{array}$ & $\mathrm{M}$ & $3 \mathrm{M}$ & 10 & 69 & 6 & $\mathrm{PCP} / \mathrm{CMV}, \mathrm{HIV}$ & 9 & 8 & 34 & Alive \\
\hline $\begin{array}{c}\text { Case } \\
5\end{array}$ & $\mathrm{~F}$ & $3 \mathrm{M}$ & 10 & 68 & 5 & $\mathrm{CMV}$ & 8 & 17 & 17 & Dead \\
\hline $\begin{array}{c}\text { Case } \\
6 \\
\end{array}$ & $\mathrm{~F}$ & $9 \mathrm{Y} \square \mathrm{M}$ & 12 & 46 & 6 & $\mathrm{VZV}, \mathrm{RV}$ & 12 & 14 & 14 & Dead \\
\hline
\end{tabular}

PRISM; Pediatric risk score for mortality, P/F ratio; $\mathrm{PaO}_{2} / \mathrm{FiO}_{2}$ ratio, DIC; disseminated intravascular coagulopathy, TLF; tracheal lavage fluid, NPA; nasopharyngeal aspiration, RSV; Respiratory syncytial virus, CMV; cytomegalovirus, TB; tuberculous, A/H1N1pdm; Influenza A, subtype H1N1pdm, PCP; pneumocystis jiroveci, HIV; human immunodeficiency virus, VZV; varicella zoster virus, RV; Rhino virus, LOS; Length of stay.

CMV*; CMV latent infection

Table 2. Changes in biomarkers of liver function and renal function before and after recombinant thrombomodulin administration 


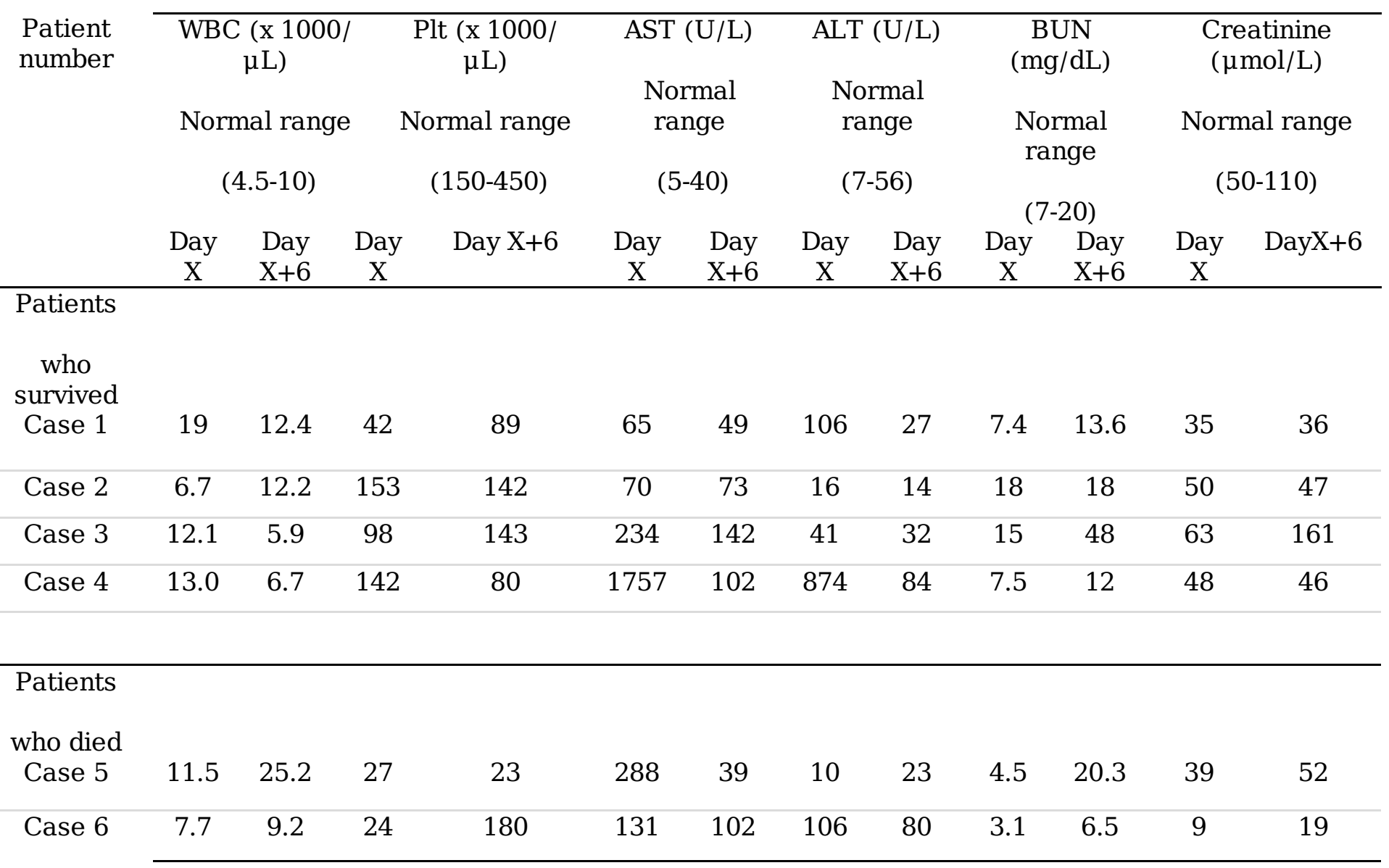

Table 3. Changes in $\mathrm{PaO}_{2} / \mathrm{FiO}_{2}$ ratio, oxygenation index and Body temperature before and after recombinant human thrombomodulin administration

\begin{tabular}{|c|c|c|c|c|c|c|c|}
\hline \multirow[t]{2}{*}{ Patient number } & \multicolumn{2}{|c|}{$\mathrm{P} / \mathrm{F}$ ratio $(\mathrm{mmHg})$} & \multicolumn{2}{|c|}{ Oxygenation Index } & \multirow{2}{*}{$\begin{array}{c}\text { Extubation } \\
\text { (day) }\end{array}$} & \multicolumn{2}{|c|}{ Body temperature } \\
\hline & Day X & Day X+6 & Day X & Day X+6 & & Day X & Day $\mathrm{X}+6$ \\
\hline \multicolumn{8}{|c|}{ Patients who survived } \\
\hline Case 1 & 100 & extubated & 15 & extubated & Yes (day X+3) & 37.0 & 37.0 \\
\hline Case 2 & 35 & 50 & 61.5 & 20 & Yes (day $\mathrm{X}+24$ ) & 38.2 & 39.7 \\
\hline Case 3 & 93 & 244 & 21 & 4.7 & Yes (day X+7) & 38.5 & 37.0 \\
\hline Case 4 & 82 & extubated & 24 & extubated & Yes (day $\mathrm{X}+4$ ) & 37.3 & 36.8 \\
\hline \multicolumn{8}{|c|}{ Patients who died } \\
\hline Case 5 & 68 & 43 & 16 & 52 & No & 38.0 & 37.7 \\
\hline Case 6 & 102 & 207 & 20 & 17 & No & 38.2 & 38.9 \\
\hline
\end{tabular}

ARDS; acute respiratory distress syndrome, $\mathrm{P} / \mathrm{F}$ ratio; $\mathrm{PaO}_{2} / \mathrm{FiO}_{2}$ ratio, LOS; Length of stay 

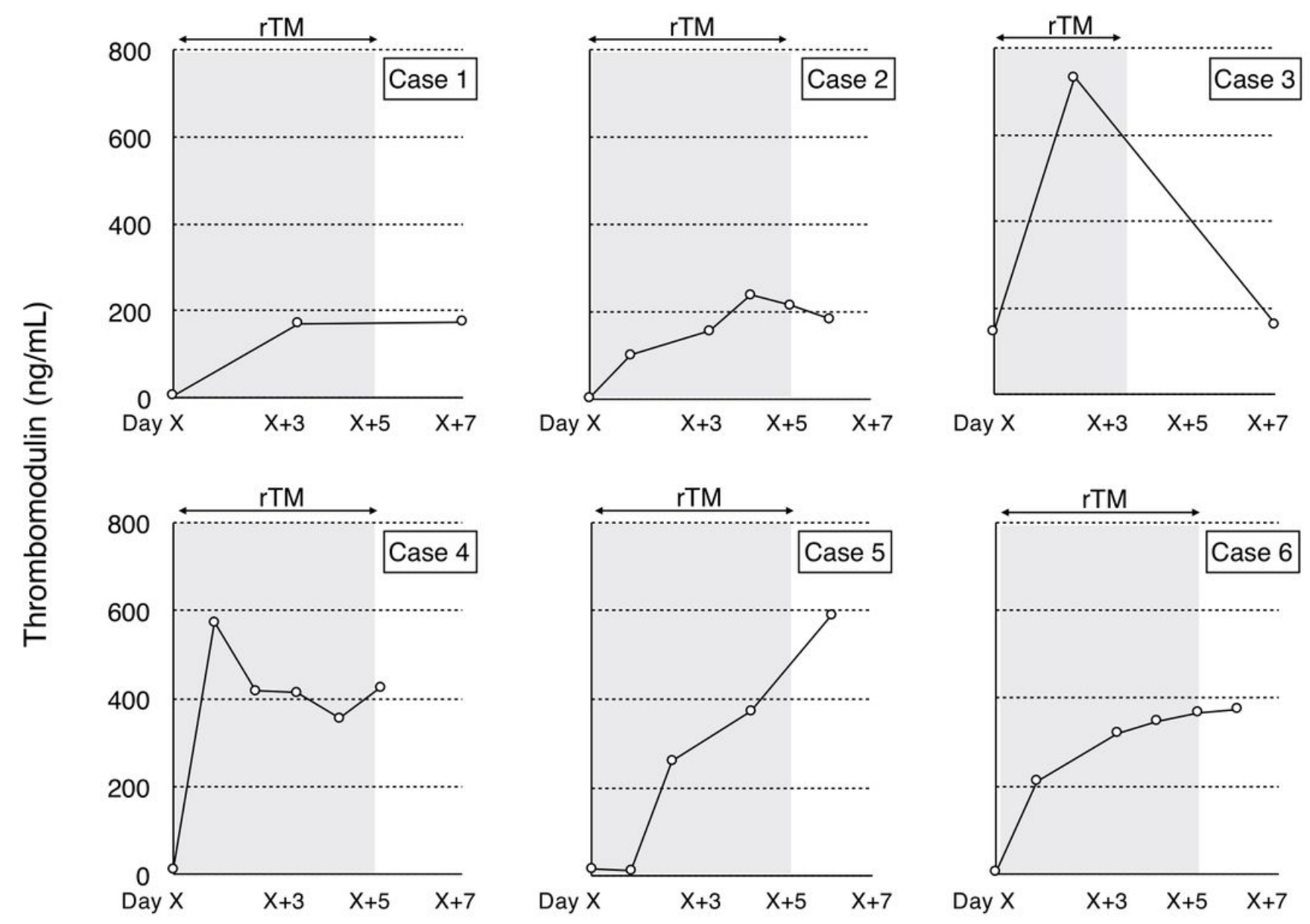

\section{Figure 1}

Changes in the plasma concentration of thrombomodulin before and after administration of recombinant human thrombomodulin (rTM). rTM administration increased the concentration of thrombomodulin in all patients. 
Figure 2

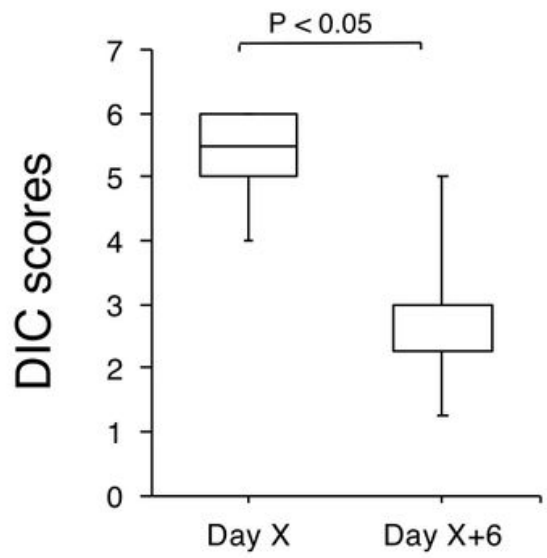

\section{Figure 2}

Changes in DIC scores before and after administration of recombinant human thrombomodulin (rTM). Data were expressed as the median and interquartile range. rTM administration significantly decreased DIC scores. 

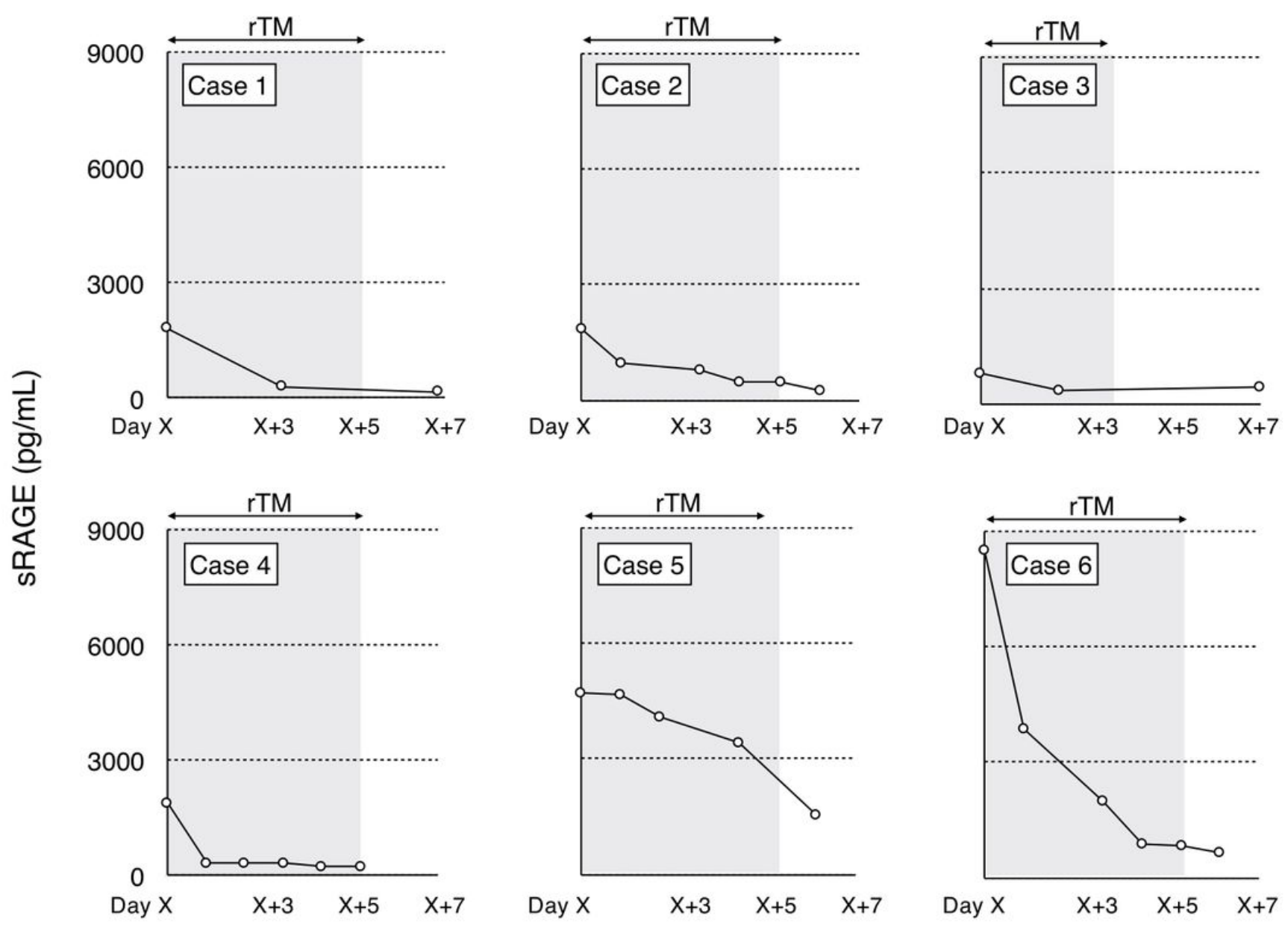

\section{Figure 3}

Changes in soluble receptor of advanced glycation end products (SRAGE) before and after administration of recombinant human thrombomodulin (rTM). rTM administration decreased sRAGE. 

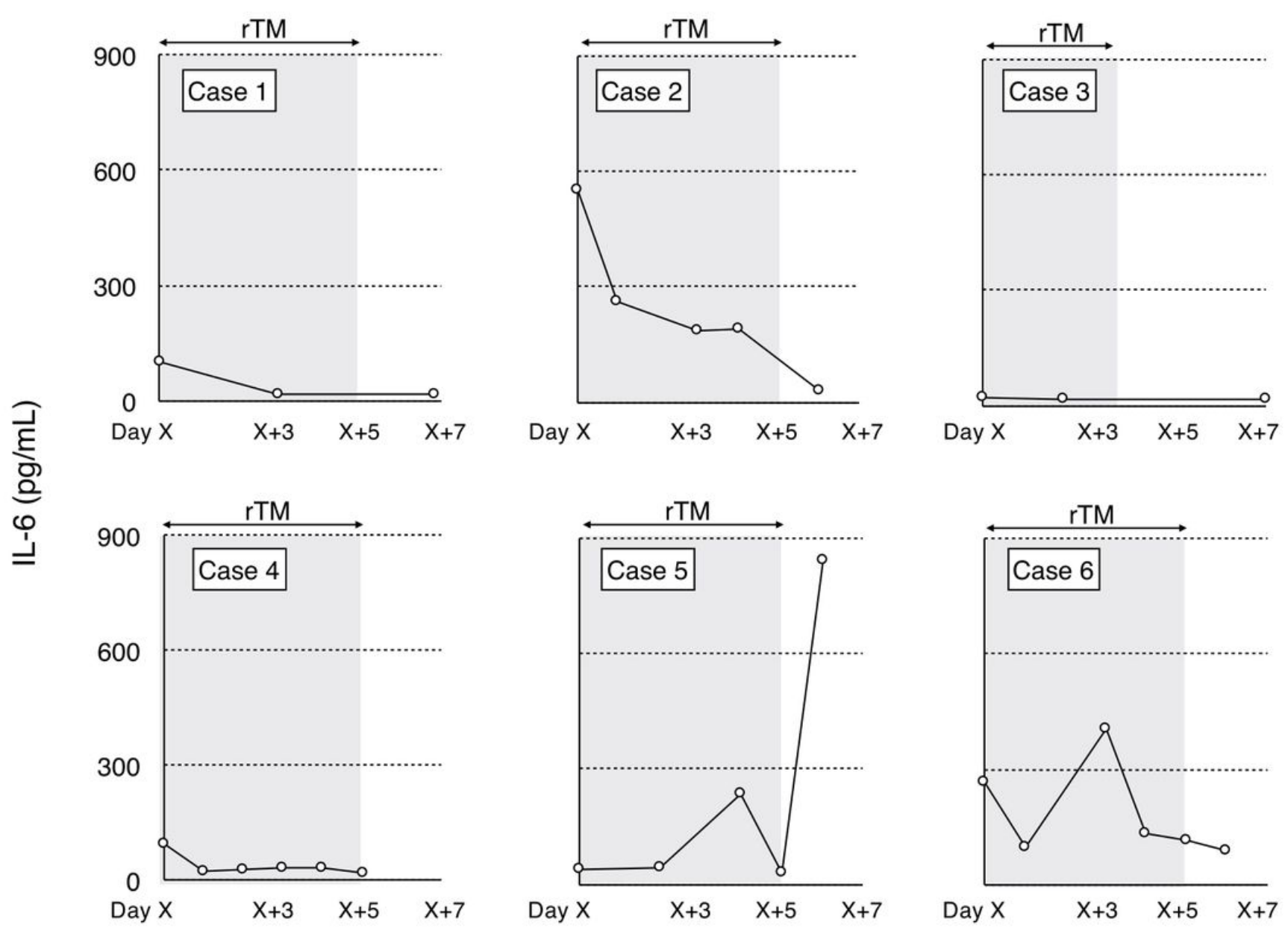

\section{Figure 4}

Changes in interleukin-6 (IL-6) before and after administration of recombinant human thrombomodulin (rTM). rTM administration significantly decreased IL-6 in the patients except case 5. 

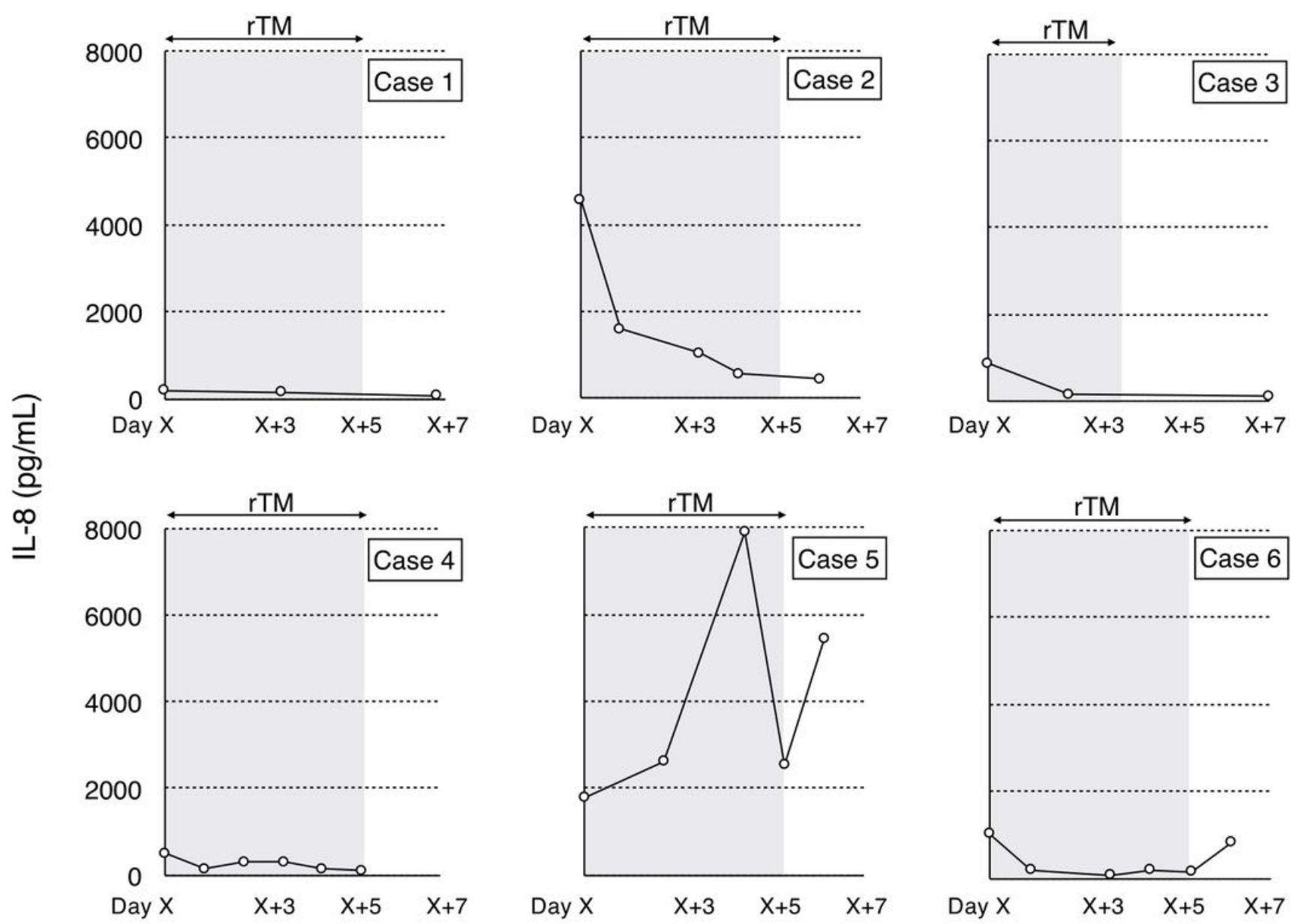

\section{Figure 5}

Changes in interleukin-8 (IL-8) before and after administration of recombinant human thrombomodulin (rTM). rTM administration significantly decreased IL-8 in the patients except case 5. 

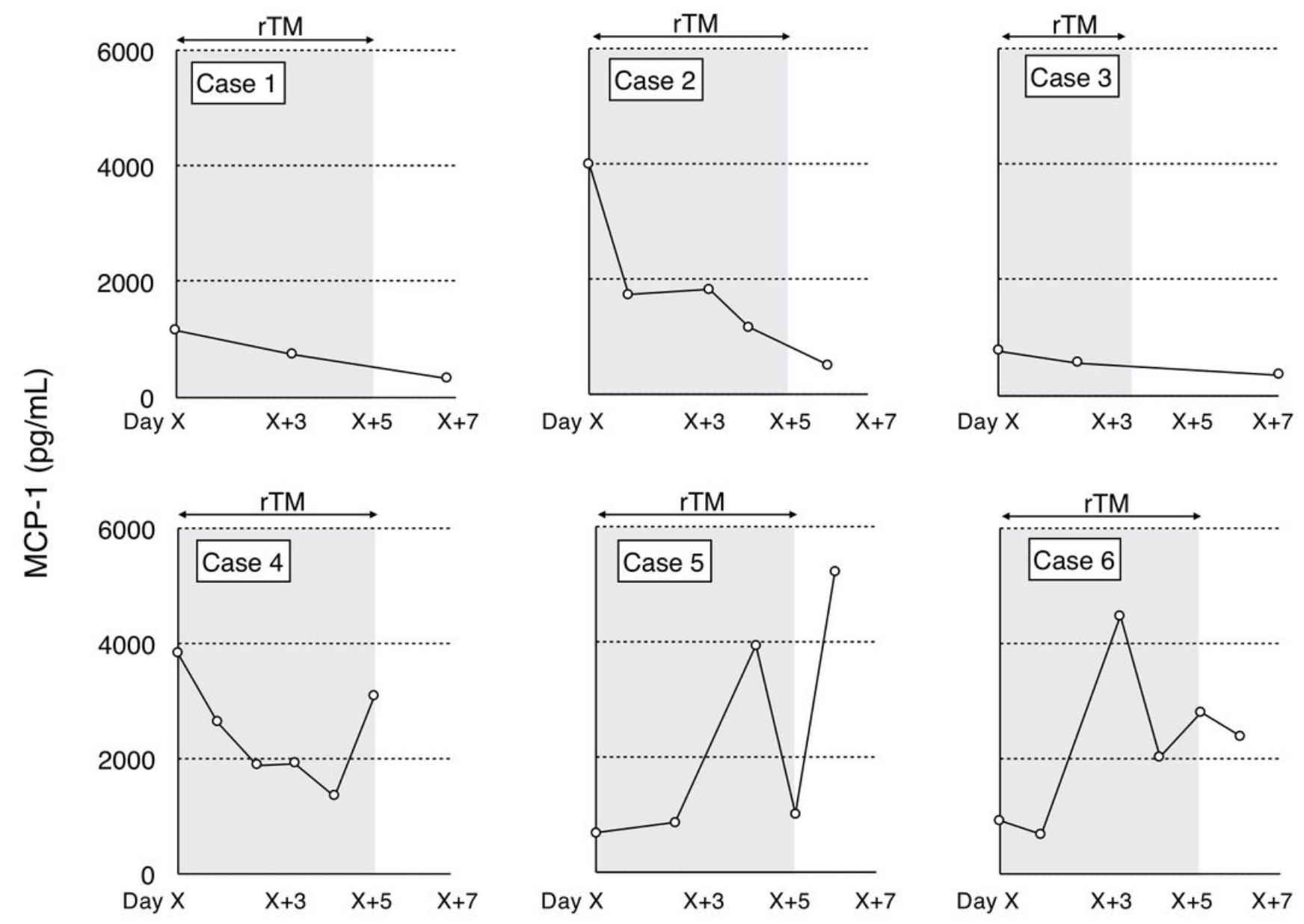

Figure 6

Changes in Monocyte chemotactic protein-1 (MCP-1) before and after administration of recombinant human thrombomodulin (rTM). rTM administration decreased MCP-1 in 3 of the 4 patients who survived, but not in patients who died.

\section{Supplementary Files}

This is a list of supplementary files associated with this preprint. Click to download.

- HirataADF1.tiff

- HirataADF4.tiff

- HirataADF5.tiff

- HirataADF3.tiff

- HirataADF6.tiff

- HirataADF2.tiff 